\title{
Biochemical metabolic modelling using fuzzy type-2
}

\author{
Zahra Shabaninia \\ Faridoon Shabaninia, Senior Member, IEEE, Shiraz University, Iran
}

Email address:

shabani@shirazu.ac.ir (Z. Shabaninia)

\section{To cite this article:}

Zahra Shabaninia. Biochemical Metabolic Modelling Using Fuzzy Type-2. American Journal of Physical Chemistry. Vol. 1, No. 1, 2012, pp. 22-25. doi: 10.11648/j.ajpc.20120101.13

\begin{abstract}
In his study a new approach, the use of fuzzy logic type-2 in modeling biochemical reactions is shown. In fact, each enzymatic reaction is modeled by means of a "sigmoid transfer function" relating input and output substrate concentrations. The slant of this function is adjusted using fuzzy type-2. This adjustment is conducted depending on the enzymatic reaction type (having activator/inhibitors or not). The obtained model seems promising in order to permit quantitative results to process data concerning adverse drugs reactions. In this paper it is also proved that by fuzzy type-2 logic, the performance characteristics of the modeling will be improved using the proposed method.
\end{abstract}

Keywords: Biochemical, Metabolic, Modeling, Fuzzy Type-2

\section{Introduction}

Biochemical metabolic chains modeling [1] is a challenging research subject. In fact, these chains are generally described as a set of substrates which are transformed, step by step by means of enzymatic reaction. Enzymatic reactions can be activated or inhibited (i.e. blocked) by specific molecules. Physical law governing these reactions is well known and it is possible, theoretically speaking, to describe a metabolic system using a set of differential equations [2]. This approach is referred to as the Numerical Modeling (NM) approach. Several difficulties remain: a) in most cases, numerical measurements for enzymatic parameter reactions are lacking or are unreliable. This is mainly due to experimental difficulties in obtaining accurate measurements of these enzymatic parameter reactions and the fact that these parameters are measured "in vitro" conditions far from "in vivo" conditions, b) most physician reports do not give quantitative clinical chemistry results but an interpretation of these results. For example, "the concentration of $\mathrm{Na}$ is too high", etc. In fact, most physicians interpret numerical measurements according to a scale that can be simply defined by the following qualitative alphabet: $\{$ "abnormally low", "normal", "abnormally high"\} describing the substrate concentration state. A Qualitative Modeling (QM) approach based on the use of fuzzy concept (type-1) and neural networks has already been proposed and tested [3-6], but no research work on fuzzy type-2 has been down yet. Obtained results have permitted to reproduce existing and known ones in a simple and a rapid way.
The organization of this paper is as follow. Section II describes the Type-2 Fuzzy Logic overview. Section III describes the Model Description and Section IV presets the conclusions.

\section{Type-2 Fuzzy Logic: Overview}

Zadeh introduced fuzzy systems in 1965 and type-2 fuzzy sets in 1975 [7]. So, after 1975, it became necessary to distinguish between pre-existing fuzzy systems and type2 fuzzy systems; hence, it became common to refer to the pre-existing FSs as "T1 FSs" and type-2 fuzzy systems as "T2 FSs" [8]. T1 FSs have been successfully used in many applications [9, 10]. However, such FSs have limited capabilities to directly handle data uncertainties, where handle means to model and minimize the effect of uncertainties.

Of course, uncertainty comes in many forms and is independent of the kind of FS or methodology one uses to handle it. Two important kinds of uncertainties are linguistic and random. The former is associated with words, and the fact that words can mean different things to different people, and the latter is associated with unpredictability. Probability theory is used to handle random uncertainty and FSs are used to handle linguistic uncertainty, and sometimes FSs can also be used to handle both kinds of uncertainty, because a fuzzy system may use noisy measurements or operate under random disturbances. Within probability theory, one begins with a probability density function (pdf) that embodies total information about random uncertainties. However, in most practical applications, it is 
impossible to know or determine the pdf; so, the fact that a pdf is completely characterized by all of its moments is used. Unfortunately, it is not possible, in practice, to determine an infinite number of moments; so, instead, at the very least, two moments are used - the mean and variance. Just as variance provides a measure of dispersion about the mean, a fuzzy set also needs some measure of dispersion to capture more about linguistic uncertainties than just a single membership function (MF), which is all that is obtained when a T1 FS is used. A T2 FS provides this measure of dispersion.

In type-2 fuzzy logic, the antecedent or consequent membership functions are type- 2 fuzzy sets. Such sets are fuzzy sets whose membership grades themselves are type-1 fuzzy sets; they are very useful in circumstances where it is difficult to determine an exact membership function for a fuzzy set; that's why it is said they are useful for incorporating uncertainties [11].

If all uncertainty disappears, then a T2 FS reduces to a T1 FS, as can be seen in Figure 1, e.g. if the uncertainties about the left- and right-end points disappear, then only the dashed triangle survives. This is similar to what happens in probability, when randomness degenerates to determinism, in which case the pdf collapses to a single point. In brief, a $\mathrm{T} 1 \mathrm{FS}$ is embedded in a T2 FS, just as determinism is embedded in randomness [12].

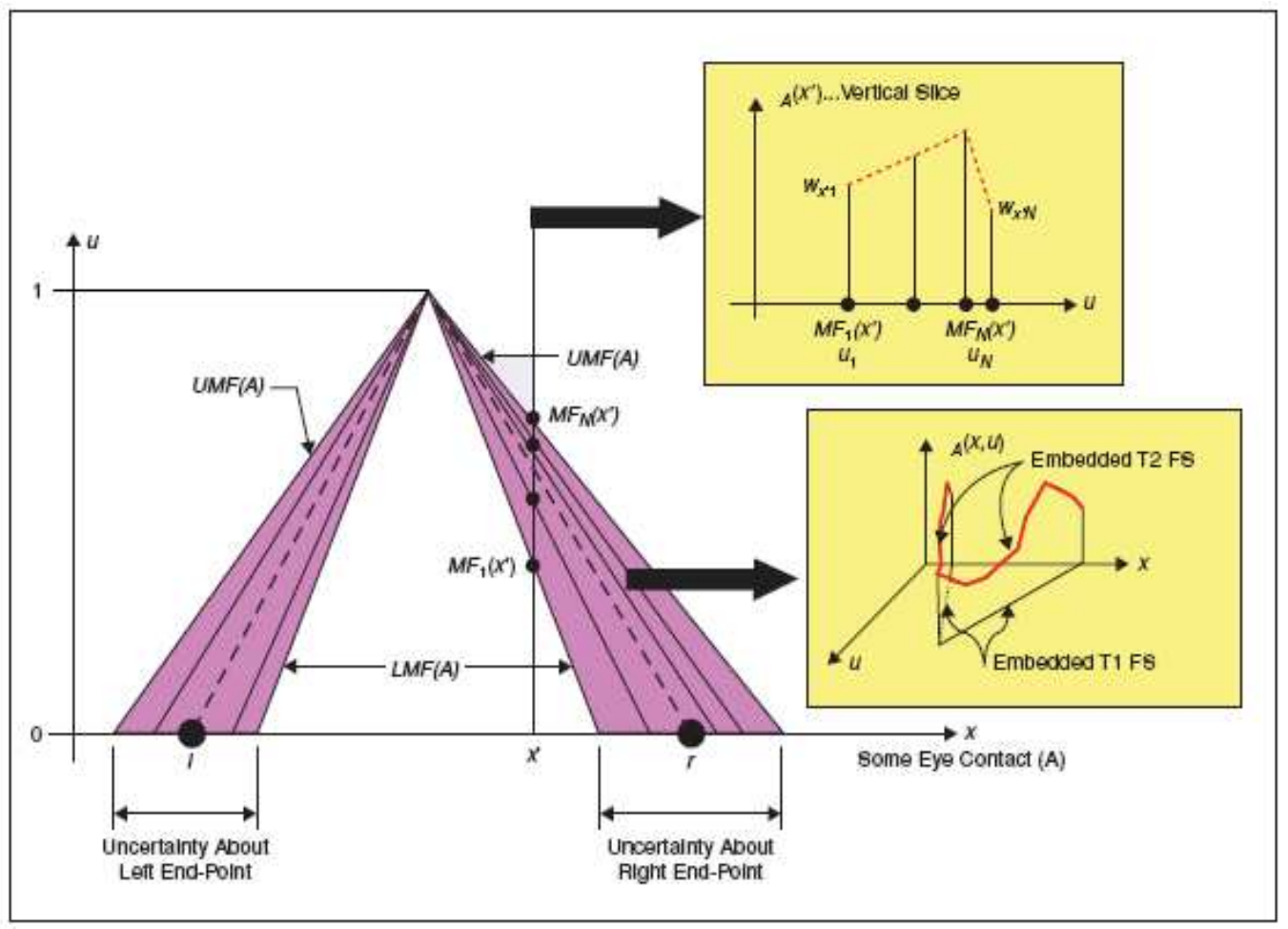

Figure 1. Triangular MFs when base end points have uncertainty intervals.

It is not as easy to sketch 3-D figures of a type-2 membership function (T2 MF). Another way to visualize a T2 FS is to sketch (plot) its footprint of uncertainty (FOU) on the 2-D domain of the T2 FS, and this is easy to do. The heights of a T2 MF (its secondary grades) sit atop its FOU.

In Figure 1. If the continuum of triangular MFs is filled in (as implied by the shading), then the FOU is obtained. Another example of an FOU is shown in Figure 2. It is for a Gaussian primary MF whose standard deviation is known with perfect certainty, but whose mean, $\mathrm{m}$, is uncertain and varies anywhere in the interval from $\mathrm{m} 1$ to $\mathrm{m} 2$. The uniform shading over the entire FOU means that uniform weighting (possibilities) is assumed. Because of the uniform weighting, this T2 FS is called an interval type-2 FS (IT2 FS) [12].

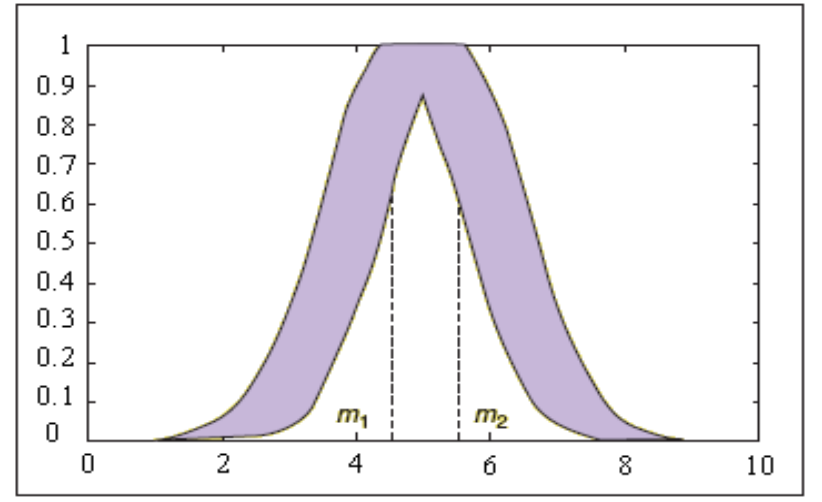

Figure 2. FOU for a Gaussian primary MF whose mean varies in the interval [m1, m2] but having constant standard deviation.

\section{Mode1 Description}


In this study, a new enzymatic reactions modeling approach is proposed. This model is based on the use of a physical reasoning method describing the enzymatic reaction (ER) functioning. Parameters updating is conducted using fuzzy type- 2 by considering the qualitative alphabet, already used, as a set of linguistic variables modeled by their membership function.

\subsection{Basic Enzymatic Reactions Model}

As previously mentioned, an enzymatic reaction (ER) permitting to transform an input substrate $S_{\text {in }}$ to output substrate $S_{\text {out }}$ is governed by a set of differential equations. The complexity of these equations is mainly due to the presence/absence of activating/inhibiting molecules as well as to the lack initial in vivo values that have to be known. Nevertheless, and whatever the complexity is, it is well known that the input and the output substrate concentrations (SC) are related together through a reaction advancement degree transfer function as shown in Figure 3.

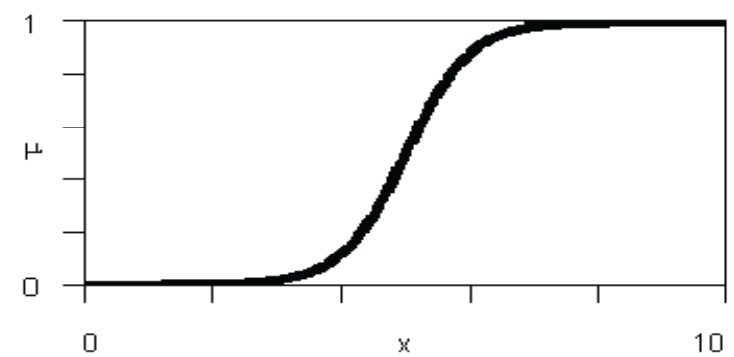

Figure 3. Reaction advancement degree.

Where: $\mathrm{X}$ represents initial input $\mathrm{SC}, \xi(\mathrm{t})$ the reaction advancement degree value and $Y(t)$ the output $\mathrm{SC}$. The analytical form of the function $\xi(\mathrm{t})$ depends strongly on the complexity of the reaction. For example, a "simple" ER with no activators neither inhibitors $\xi(t)$ is given by:

$$
\begin{gathered}
\xi(\mathrm{t})=\mathrm{Y}(\mathrm{t}) / \mathrm{X}_{0} \\
\xi(\mathrm{t})=\mathrm{KX}_{0}^{2} \mathrm{t} /\left(1+\mathrm{KX}_{0}\right) \text { for } \mathrm{t} \epsilon\left[0, \mathrm{t}_{1}\right] \\
=\mathrm{X}_{0}(1-\mathrm{e}-\mathrm{Kt}) \text { for } \mathrm{t} \epsilon\left[\mathrm{t}_{1}, \mathrm{t}_{2}\right]
\end{gathered}
$$

Where $\mathrm{K}$ is called the Michaelis-Menten parameter. Given the fact that an input substrate can be transformed through several ER, as well as the basic analytic forms of the function $\xi(\mathrm{t})$ depend strongly on the activating and inhibiting substrate concentrations, an exact analytical modeling of such reaction becomes extremely complex. In the proposed model, all the analytic components that may compose the function $\xi(\mathrm{t})$ are reduced to a single sigmoid transfer function in which the slant is adapted in a dynamic way in order to take into account the dynamic behavior of different SCs.

\subsection{Substrate Concentration Description}

In the proposed model, the substrate concentrations (SC) are described using the qualitative alphabet: Abnormally low (AL), normal $(\mathrm{N})$ and abnormally high $(\mathrm{AH})$. This description is modified through the use of fuzzy type-2. In fact, the fuzzification is conducted by considering the three elements of the alphabet as linguistic variables modeled through the use of three membership functions and each input is assigned a boundary membership grade (Figure 4).

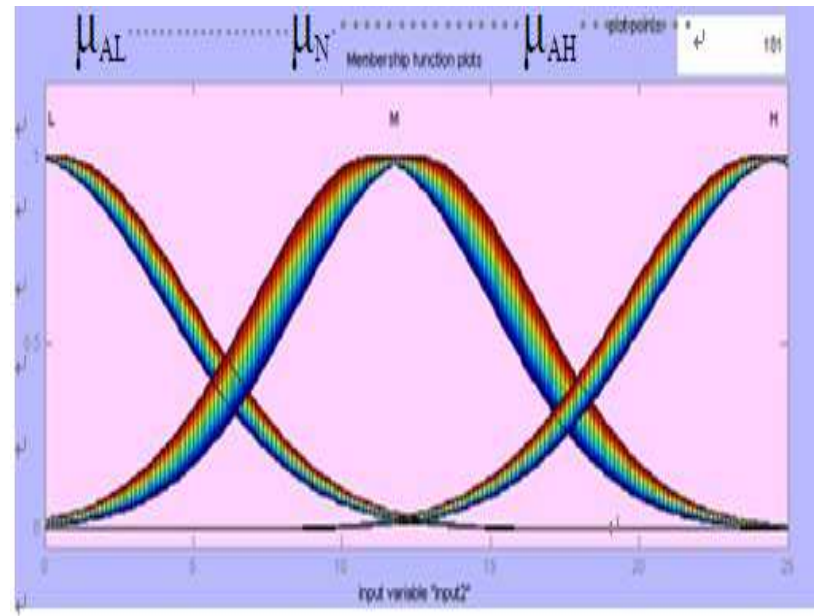

Figure 4. Linguistic substrate concentration variables.

Therefore, a SC " $\mathrm{x}$ " is considered through a membership vector: $\mu=\left(\mu_{\mathrm{AL}}, \mu_{\mathrm{N}}, \mu_{\mathrm{AH}}\right.$, $)$ instead of single value.

\subsection{Slants Dynamic Adaptation}

In order to describe the proposed method of slant adaptation, the following configurations are discussed:

\subsubsection{Basic ER}

The term "basic" denotes enzymatic reactions having neither activating nor inhibiting substrates. In this case, the reaction is in the "active" state all the time and the sigmoid transfer function is determined through a fixed slant. This representation has the important advantage of discriminating between the so called normal and major reactions through the selection of different slant values.

\subsubsection{ER with Activating Substrates}

These ER is governed by the activating molecules. In the qualitative modeling approach, the ER is considered as "blocked" when the activating molecules concentration is abnormally low. In this case, the product concentration is considered as abnormally low and the input SC abnormally high. Otherwise, the QM approach "duplicates" the input SC into the output product concentration. This simple reasoning has shown to be very attractive [3] through its simplicity as well as the equivalence between obtained simulation results with those already described in the specialized literature. In the proposed model, a similar reasoning method is adopted. In fact, as it is shown on Figure 6, the QM is obtained as the extreme cases of this model: Slant $=0$ for the blocked ER state and, Slant=Smax=1 for the activated ER state. 


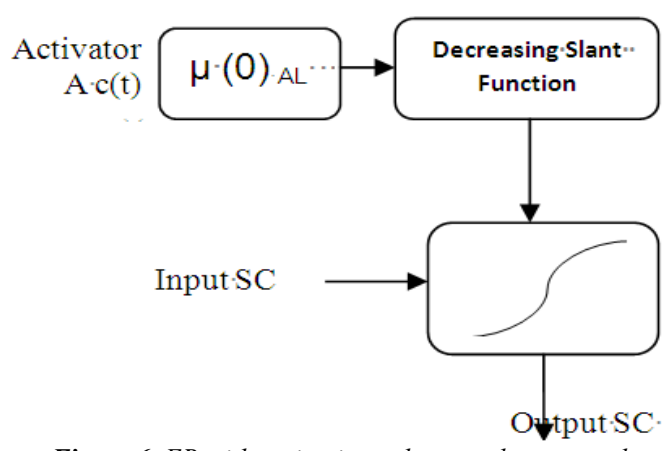

Figure 6. ER with activating substrate slant control.

The first advantage of this model is that it takes into account the continuous substrate production through an important parameter, generally ignored in QM, the time parameter.

\subsubsection{ER with Inhibiting Substrates}

These ER is governed by the inhibiting molecules concentration. In the QM approach, the ER is considered as "blocked" when the inhibiting molecules concentration is abnormally high. This type of ER is modeled similarly to the previous case by substituting $\mu \mathrm{AL}$ by $\mu \mathrm{AH}$ applied into the inhibiting molecules concentration.

\subsubsection{ER with Activating and Inhibiting Substrates}

These ER are governed by the ration $\mathrm{R}=$ Activating molecules concentration / inhibiting molecules concentration. When $\mathrm{R}>1$ the ER is considered in the activated state. Otherwise, the ER is considered as in the blocked state. In this case, the slant updating is based on the following function (Figure 5).

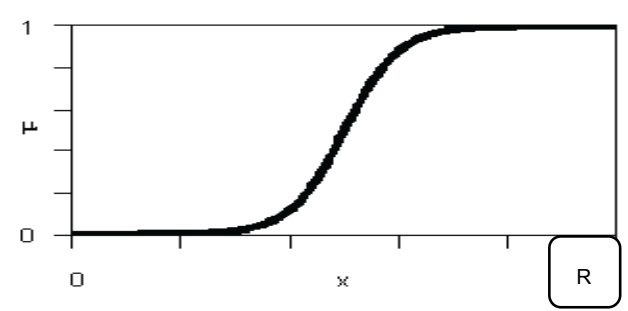

Figure 5. Slant updating for ER with activating and inhibiting substrates.

\section{Conclution}

In this study, a new promising approach using fuzzy type- 2 is proposed in modeling enzymatic reactions. In fact, the common use of the NM (sigmoid transfer function) and QM (linguistic variables controlling the sigmoid slant values) has permitted to resolve the following difficulties:

a) major/normal production pathways through varying slant values,

b) modeling all ER using a unified approach, Remind that in this study, ER are considered as independent entities and the efforts were oriented towards their modeling. In reality, even a simple metabolic chain contains at least several dozen of ER for which different substrates can be activators, inhibitors or input substrates. Therefore, actual efforts are mainly conducted in order to put in cooperation, for a specific metabolic chain (like the pure bases biosynthesis one), several ER fuzzy type-2 representation and then to evaluate the global obtained system. The proposed architecture utilizes the capabilities of a type-2 fuzzy architecture in properly handling of uncertainties in computing the output. Two main criteria are essential in such evaluation: the fact of obtaining an equilibrium steady state after the introduction of a perturbation and the recovery of clinical observations related to genetic diseases (i.e. disfunctionning of specific ER).

\section{References}

[1] G.B. Grindey and Y.C. Cheng, "Biochemical and kinetic approaches to inhibition of multiple pathways". Pharmacy and therapy, 4, pp.307-327, 1979.

[2] C. Reder, "Metabolic control theory", Journal of Theoretical Biology, 135, pp. 175-201, 1988.

[3] B. Solaiman and D. Picart, "Neural networks modeling of biochemical reactions", IEEE Intenational Conference on Neural Networks, ICNN94, June 26-Jul 2, Orlando, USA 1994.

[4] G. F. Cerofolini and P. Amato, "Fuzzy Chemistry-An Axiomatic Theory for General Chemistry", IEEE 2007.

[5] Christian Wagner and Simon Miller, "A Fuzzy Toolbox for the R Programming Language", IEEE 2011.

[6] Xiang Li, Xin Lu, Jing Tian,, Peng Gao, Hongwei Kong, and Guowang $\mathrm{Xu}^{*}, \dagger$ Application of Fuzzy c-Means Clustering in Data Analysis of Metabolomics", Anal. Chem. 2009, 81, 4468-447.

[7] L.A. Zadeh, "The concept of a linguistic variable and its application to approximate reasoning-1", Information Sciences, vol. 8, pp. 199-249, 1975.

[8] J.M. Mendel, "Uncertain Rule-Based Fuzzy Logic Systems: Introduction and New Directions", Prentice-Hall, UpperSaddle River, NJ, 2001.

[9] F. Shabaninia, "Type-2 Fuzzy Multiagent Traffic Signal Control", IEEE-IRI Conference, Las Vegas 2012.

[10] F. Shabaninia, "Fuzzy Type-2 Electrode Position Controls for an Electric Arc Furnace ", IEEE-IRI Conference, Las Vegas 2012.

[11] J. M. Mendel, "Type-2 Fuzzy Sets and Systems: An Overview”, IEEE Computational Intelligence Magazine, 2007.

[12] N. N. Karnik, J. M. Mendel, Q. Liang, "Type-2 Fuzzy Logic Systems", IEEE Transactions on Fuzzy Systems, vol. 7, NO. 6, 1999. 